\title{
ON THE REAL MOMENT MAP
}

\author{
Alina Marian
}

\section{Introduction}

This paper presents an extension of a theorem, due to Ness ([5], Theorem 6.2), to the case of real group actions. Ness considers a continuous representation of a complex reductive group $G$ on a complex finite-dimensional vector space $V$. If $\mathfrak{k}$ is the Lie algebra of a maximal compact subgroup $K$ of $G$ and $\mathfrak{g}=\mathfrak{k} \oplus i \mathfrak{k}$ is the Lie algebra of $G$, then upon fixing a $K$-invariant Hermitian inner product on $V$, a map $m: \mathbb{C P}(V) \longrightarrow i \mathfrak{k}^{\star}$ is defined as follows: for $\beta \in i \mathfrak{k}$ and $v \in V$ representing $x \in \mathbb{C P}(V),\langle m(x), \beta\rangle=_{\text {def }} \frac{(\beta v, v)}{\|v\|^{2}}$. Fix an $A d K$-invariant inner product on $i \mathfrak{k}^{\star}$, and let $\|m\|^{2}$ denote the squared norm of $m,\|m\|^{2}: \mathbb{C P}(V) \longrightarrow \mathbb{R}$, $\|m\|^{2}(x)=\|m(x)\|^{2}$. Ness's result concerns the critical points of $\|m\|^{2}$, and it states that 1 . If $x$ is a critical point of $\|m\|^{2}$, then $\left.\|m\|^{2}\right|_{G x}$ achieves a minimum at $x$. 2. If nonempty, the critical set of $\|m\|^{2}$ contained in a $G$-orbit $G x$ consists exactly of a $K$-orbit, and is in particular connected.

The present paper takes note of the fact that the same connectedness statement about critical points in a $G$-orbit holds in the case of actions of real semisimple Lie groups on real vector spaces.

The context of [5] is related to the situation of a compact group $K$ 's action on a symplectic manifold $X$, such that the action admits a moment map $m: X \longrightarrow \mathfrak{k}^{\star}$. Indeed, if the map $m: \mathbb{C P}(V) \longrightarrow i \mathfrak{k}^{\star}$ is given as before, then $\tilde{m}: \mathbb{C P}(V) \longrightarrow \mathfrak{k}^{\star}$, defined by $\tilde{m}(x)(\beta)=m(x)(i \beta)$, is a moment map with respect to the action of $K$ and the Kähler structure on $\mathbb{C P}(V)$ induced by the Hermitian structure on $V$. The result of [5], as it stands, bears on problems in geometric invariant theory: trying to understand, with suitable modifications, the $G$-orbit space on $\mathbb{C P}(V)$ as an algebraic variety. Transposed in the context of real groups, Ness's statement has mostly representation-theoretic utility. It eases, for instance, the geometric understanding of bijective relationships between certain types of orbits arising in the situation of real semisimple group actions. The geometric interpretation is obtained by considering the stratification of a subvariety $X$ of $\mathbb{P}(V)$ which is acted on by a real semisimple group, induced by the gradient flow of the function $\|m\|^{2}$. Each stratum is associated with a connected component of the critical set: it consists of the points of $X$ whose grad $\|m\|^{2}$ trajectory

Received September 25, 2001. 
has its limit points in that component of the critical set. The correspondence then results by realizing the two different types of orbits - between which one wishes to establish the correspondence - as strata of two distinct moment map stratifications with the same critical set; orbits thus get associated to one another via the connected components of the critical set. This idea is carried out in the case of the Sekiguchi and Matsuki correspondences in [7] and [3] respectively.

The real setting is given by a real semisimple group $G$, with a Cartan decomposition $\mathfrak{g}=\mathfrak{k} \oplus \mathfrak{p}$, acting on real projective space $\mathbb{P}(V)$ by a representation of $G$ on $V$, and $m: \mathbb{P}(V) \longrightarrow \mathfrak{p}^{\star}$ defined as before. The statement is that if nonempty, the set of critical points of $\|m\|^{2}: \mathbb{P}(V) \rightarrow \mathbb{R}$ in a $G$-orbit consists of a unique $K$ orbit. The argument follows structurally that given by Ness ([5], [6]) in the case of a complex group action: all the intermediate statements are retained from [5] in a simplified succession, as well as some of their proofs. The most important intermediate step establishes the fact that $\|m\|^{2}$ assumes the same value at critical points in one $G$-orbit. Ness invokes two distinct statements which together would imply it. One is the assertion that a certain function $M: \mathbb{C P}(V) \longrightarrow \mathbb{R}$ introduced by Mumford ([4]) to characterize stability of vectors in $V$ relative to the action of $G$, is $G$-invariant. The second one is the convexity property of $m$ established by Guillemin and Sternberg ([1]). It is however easy to see that the convexity property is not needed, and that in order to prove the result, the $G$-invariance of $M$ alone suffices. The argument given here for the real setting proceeds by showing the $G$-invariance of Mumford's function for $G$ real semisimple, in a manner inspired by [6], and it relies entirely on basic facts about the structure of real semisimple Lie groups. This is the original core of the paper, and is presented in Section 3. Serving as background, Section 2 records well-known facts, some of which are used in the subsequent part.

\section{Preliminaries}

Our setting will be the linear action of a real semisimple Lie group on a real finite dimensional vector space. We shall regard it as an action on the corresponding projectivized space $\mathbb{P}(V)$. In this situation, upon choosing an inner product on the vector space we introduce a map $m$ that associates to each point in the projectivized space the rate of the squared-norm variation of one of its unit representatives, along the group. Specifically, let $G$ be the group, and let $\Phi: G \longrightarrow G L(V)$ denote the continuous representation giving the action. The Lie algebra $\mathfrak{g}$ of $G$ acts linearly also, by $d \Phi_{e}: \mathfrak{g} \longrightarrow \mathfrak{g l}(V)$. Let $\mathfrak{g}=\mathfrak{k}+\mathfrak{p}$ be a Cartan decomposition of the algebra $\mathfrak{g}$ and let $():, V \times V \longrightarrow \mathbb{R}$ be an inner product compatible with this Cartan decomposition i.e., such that when $X \in \mathfrak{k}, Y \in \mathfrak{p}, X, Y: V \longrightarrow V$ are respectively antisymmetric and symmetric relative to $($,$) . Define \psi_{v}: G \longrightarrow \mathbb{R}$ by $\psi_{v}(g)=\|g v\|^{2}$ and $m: \mathbb{P}(V) \longrightarrow \mathfrak{g}^{\star}$ by $m(x)=\frac{1}{2\|v\|^{2}}\left(d \psi_{v}\right)_{e}$, where $v \in V$ is a representative of $x \in \mathbb{P}(V)$. Let $K$ be the maximal compact subgroup of $G$ with algebra $\mathfrak{k}$. The inner product on $V$ has been chosen to be $K$-invariant, thus $\left(d \psi_{v}\right)_{e}$ is identically 0 on $\mathfrak{k}$. Also upon 
fixing an $A d(K)$-invariant inner product on $\mathfrak{p},(,)_{\mathfrak{p}}: \mathfrak{p} \times \mathfrak{p} \longrightarrow \mathbb{R}$, and thereby identifying $\mathfrak{p}$ and $\mathfrak{p}^{\star}, m$ becomes a map from $\mathbb{P}(V)$ to $\mathfrak{p}, m: \mathbb{P}(V) \longrightarrow \mathfrak{p}$.

Note that for $\beta \in \mathfrak{p}$,

$$
(m(x), \beta)_{\mathfrak{p}}=\left.\frac{1}{2\|w\|^{2}} \frac{d}{d t}\right|_{t=0}\|\exp t \beta w\|^{2}=\frac{(\beta w, w)}{\|w\|^{2}},
$$

where $w$ is a representative of $x$.

In order to place the map $m$ into perspective, we briefly abandon the situation that strictly interests us, the linear action of a real semisimple group, and try to do justice to a statement in the introduction - that $m$ is a real version of the moment map on complex projective space. This is not so much useful for the subsequent presentation, but it is rather viewed as a cultural demarche. Thus if instead of dealing with the case of a noncompact semisimple group acting on real projective space, we were in the context of a compact group $K$ acting on complex projective space via a continuous representation, upon choosing $K$ and $A d K$ invariant inner products on $V$ and $\mathfrak{k}$ respectively, we would have the following

Proposition 1. The map $m: \mathbb{C P}(V) \longrightarrow \mathfrak{k}$ defined by $(m(x), \beta)_{\mathfrak{k}}=\frac{1}{2 \pi i} \frac{(\beta w, w)}{\|w\|^{2}}$, $\beta \in \mathfrak{k}$ and $w$ over $x$, is the moment map associated with the natural symplectic structure of complex projective space, coming from the Fubini-Study metric, and with the action of the compact group $K$ on $\mathbb{C P}(V)$.

Proof. Recall that given a symplectic manifold $(X, \omega)$ and a smooth action of a compact group $K$ on $X$ which preserves the symplectic form, a map $m: X \longrightarrow \mathfrak{k}$ is a moment map for the action if the following two conditions are satisfied:

1. $m$ is equivariant relative to the adjoint action on $\mathfrak{k}: \forall k \in K, \forall x \in X, m(k x)=$ $\operatorname{Adk}(m(x))$.

2. For $\beta \in \mathfrak{k}$, denote by $m_{\beta}(x)$ the value of $m(x)$ in the direction of $\beta$, $m_{\beta}: X \longrightarrow \mathbb{R}, m_{\beta}(x)=(m(x), \beta)$. Let $x \mapsto \hat{\beta}_{x}$ be the vector field on $X$ generated by $\beta$ : $\hat{\beta}_{x}=\left.\frac{d}{d t}\right|_{t=0} \exp t \beta x$. Then $m_{\beta}$ is a Hamiltonian function for the vector field $x \mapsto \hat{\beta}_{x}$ i.e., for all $\zeta \in T_{x} X,\left\langle d m_{\beta}(x), \zeta\right\rangle=\omega_{x}\left(\zeta, \hat{\beta}_{x}\right)$.

It is clear that once a moment map exists, the condition $\left\langle d m_{\beta}(x), \zeta\right\rangle=$ $\omega_{x}\left(\zeta, \hat{\beta}_{x}\right)$ ensures that it is also unique up to an additive constant.

Now $K$-equivariance of $m$ defined as in Proposition 1 is immediate: $\begin{aligned}(m(k x), \beta)_{\mathfrak{k}} & =\frac{1}{2 \pi i} \frac{\left(k^{-1} \beta k w, w\right)}{\|w\|^{2}}=\frac{1}{2 \pi i} \frac{\left(A d k^{-1}(\beta) w, w\right)}{\|w\|^{2}} \\ & =\left(m(x), A d k^{-1}(\beta)\right)_{\mathfrak{k}}=(A d k(m(x)), \beta)_{\mathfrak{k}} .\end{aligned}$

To show the second moment map property of $m$, recall that the Fubini-Study metric is a Hermitian metric on $T \mathbb{C P}(V)$ defined as follows. Let $($,$) be a Her-$ mitian inner product on $V$ preserving $K$. Let $\Pi: V-\{0\} \longrightarrow \mathbb{C P}(V)$ be the 
standard projection. For $\zeta_{1}, \zeta_{2} \in T_{x} \mathbb{C P}(V)$ and $\xi_{1}, \xi_{2} \in T_{w} V$, w over $x$, such that $\Pi_{\star}\left(\xi_{i}\right)=\zeta_{i}, 1 \leq i \leq 2$, one sets $\left(\zeta_{1}, \zeta_{2}\right)_{F S}=\frac{\left(\xi_{1}, \xi_{2}\right)(w, w)-\left(\xi_{1}, w\right)\left(w, \xi_{2}\right)}{(w, w)^{2}}$. It is immediate that $\Pi$ is a submersion and that for every $w$ in $V$, $\operatorname{ker}\left(\Pi_{\star}\right)_{w}=\operatorname{Span}\{w\}$. Using the inner product on $V$, for $w$ over $x$ we can thus identify $T_{x} \mathbb{C P}(V)$ with the orthogonal complement of $w$ in $V, T_{x} \mathbb{C P}(V) \simeq \operatorname{Span}\{w\}^{\perp}$. The Hermitian metric simplifies then to $\left(\zeta_{1}, \zeta_{2}\right)_{F S}=\frac{\left(\zeta_{1}, \zeta_{2}\right)}{(w, w)}$. The symplectic structure is provided by its imaginary part, $\omega\left(\zeta_{1}, \zeta_{2}\right)=-\frac{1}{\pi} \operatorname{Im}\left(\zeta_{1}, \zeta_{2}\right)_{F S}$.

For $\beta \in \mathfrak{k}$, the vector field $x \mapsto \hat{\beta}_{x}$ is given by $\hat{\beta}_{x}=\left.\frac{d}{d t}\right|_{t=0} \exp t \beta x=$ $\Pi_{\star}\left(\beta_{w}\right)$, where $\beta_{w}=\left.\frac{d}{d t}\right|_{t=0} \exp t \beta w=\beta w$. Under the identification $T_{x} \mathbb{C P}(V) \simeq$ $\operatorname{Span}\{w\}^{\perp}, \hat{\beta}_{x}=\Pi_{\star}(\beta w)$ thus corresponds to the vector $\beta w-\frac{(\beta w, w)}{(w, w)} w$ and for $\zeta$ in $\operatorname{Span}\{w\}^{\perp}, \omega\left(\zeta, \hat{\beta}_{x}\right)=-\frac{1}{\pi} \operatorname{Im}\left(\zeta, \beta w-\frac{(\beta w, w)}{(w, w)} w\right)_{F S}=-\frac{1}{\pi} \operatorname{Im} \frac{(\zeta, \beta w)}{(w, w)}=$ $\frac{1}{\pi} \operatorname{Im} \frac{(\beta w, \zeta)}{(w, w)}$.

On the other hand, since $m_{\beta}(x)=\frac{1}{2 \pi i} \frac{(\beta w, w)}{(w, w)}$, we have, for $\zeta \in \operatorname{Span}\{w\}^{\perp}$, $\left\langle d m_{\beta}(x), \zeta\right\rangle=$

$\left.\frac{1}{2 \pi i} \frac{d}{d t}\right|_{t=0} \frac{(\beta(w+t \zeta), w+t \zeta)}{(w+t \zeta, w+t \zeta)}=\frac{1}{2 \pi i} \frac{(\beta w, \zeta)+(\beta \zeta, w)}{(w, w)}$. As $\beta: V \longrightarrow V$ is antisymmetric, the last quantity equals $\frac{1}{\pi} \frac{\operatorname{Im}(\beta w, \zeta)}{(w, w)}$, hence $\left\langle d m_{\beta}(x), \zeta\right\rangle=\omega\left(\zeta, \hat{\beta}_{x}\right)$, as desired.

Let us now go back to the situation of interest to us, real actions of semisimple noncompact groups, as set up in the first paragraph of this section. For $\beta$ in $\mathfrak{p}$, in this case also denote by $m_{\beta}(x)$ the component of $m(x)$ in the direction of $\beta$. For $w \in V$ over $x$, the same realization of $T_{x} \mathbb{P}(V)$ as $\operatorname{Span}\{w\}^{\perp} \subset V$ is convenient. The Fubini-Study metric provides $\mathbb{P}(V)$ with a Riemannian (instead of a Hermitian) structure. As in eq. (1), $m_{\beta}(x)=(m(x), \beta)_{\mathfrak{p}}=\frac{(\beta w, w)}{(w, w)}$, thus $\left\langle d m_{\beta}(x), \zeta\right\rangle=\left.\frac{d}{d t}\right|_{t=0} \frac{(\beta(w+t \zeta), w+t \zeta)}{(w+t \zeta, w+t \zeta)}=\frac{(\beta w, \zeta)+(\beta \zeta, w)}{(w, w)} . \beta \in \mathfrak{p}$ is symmetric with respect to $($,$) , the real inner product on V$, so

$$
\left\langle d m_{\beta}(x), \zeta\right\rangle=\frac{2(\beta w, \zeta)}{(w, w)}=2\left(\hat{\beta}_{x}, \zeta\right)_{F S}
$$

We have obtained the following

Lemma 1. The gradient of $m_{\beta}$ with respect to the Fubini-Study metric is $\left(\operatorname{gradm} m_{\beta}\right)_{x}=2 \hat{\beta}_{x}$. In particular, $\left(\operatorname{grad} m_{\beta}\right)_{x}=0$ if and only if $\hat{\beta}_{x}=0$ if and only if $\left.\frac{d}{d t}\right|_{t=0} \exp t \beta x=0$ if and only if $x$ is fixed by $\exp t \beta$.

The function that we shall be working with is the squared norm of the real moment map $m,\|m\|^{2}: \mathbb{P}(V) \longrightarrow \mathbb{R},\|m\|^{2}(x)=\|m(x)\|_{\mathfrak{p}}^{2}$. We are interested in the critical set of this function, which the following lemma helps to characterize. 
Lemma 2. Let grad $\|m\|^{2}$ denote the gradient of $\|m\|^{2}$ with respect to the FubiniStudy metric. Then $\left(\operatorname{grad}\|m\|^{2}\right)_{x}=4 \widehat{m(x)}_{x}$. Therefore $x$ is a critical point of $\|m\|^{2}$ if and only if the vector field $\widehat{m(x)} \subset T \mathbb{P}(V)$ induced by $m(x) \in \mathfrak{p}$ vanishes at $x$, if and only if $\exp \operatorname{tm}(x)$ fixes $x$.

Proof Let $\beta_{1}, \ldots, \beta_{n}$ be an orthonormal basis of $\mathfrak{p}$. Then $m(x)=\sum_{i} m_{\beta_{i}}(x) \beta_{i}$ and $\|m\|^{2}=\sum_{i} m_{\beta_{i}}^{2}$. Hence $\left\langle d\|m\|^{2}, \zeta\right\rangle=2 \sum_{i} m_{\beta_{i}}\left\langle d m_{\beta_{i}}, \zeta\right\rangle$

$\left.=2 \sum_{i} m_{\beta_{i}}\left(\operatorname{grad} m_{\beta_{i}}, \zeta\right)_{F S}=4 \sum_{i} m_{\beta_{i}} \frac{\left(\beta_{i} w, \zeta\right)}{(w, w)}=4 \frac{(m(x) w, \zeta)}{(w, w)}=4(\widehat{m(x)})_{x}, \zeta\right)_{F S}$.

\section{The Real Version of Ness's Theorem}

Recall our setting. $G$ is a real semisimple Lie group with the Cartandecomposed algebra $\mathfrak{g}=\mathfrak{k} \oplus \mathfrak{p} . m: \mathbb{P}(V) \longrightarrow \mathfrak{p}$ is defined as before, $(m(x), \beta)_{\mathfrak{p}}=$ $\frac{(\beta v, v)}{(v, v)}$. From now on we shall drop the subscript $\mathfrak{p}$ and shall write $(m(x), \beta)$ for $(m(x), \beta)_{\mathfrak{p}}$. We wish to prove

Theorem 1. 1) If $x$ is a critical point of $\|m\|^{2}$, then $\left.\|m\|^{2}\right|_{G x}$ achieves a minimum at $x$.

2)If nonempty, the critical set of $\|m\|^{2}$ contained in a $G$-orbit consists of a unique K-orbit.

Proof. Part 2 is harder to show than Part 1. The main step in the proof of 2 is showing that for $x$ and $y$ critical points in the same $G$-orbit, $\|m\|^{2}(x)=\|m\|^{2}(y)$. Part 1 will be proved on the way toward this result.

To start, let us consider the (easier to deal with) case when $x$ is a critical point of $\|m\|^{2}$ such that $m(x)=0$. It is easy to argue directly (and we shall do it) that $m$ vanishes in this case on the entire critical subset of $\|m\|^{2}$ contained in the orbit $\mathcal{O}=G x$ of $x$. This would establish in the zero-value situation the claim that $\|m\|^{2}$ assumes the same value at critical points in the same $G$-orbit. Note that the first part of the theorem is true in this case since $\|m\|^{2}$ is by definition nonnegative. Suppose therefore that $y \in \mathcal{O}$ is such that $y$ is critical but $m(y) \neq 0$. Let $\mathfrak{a}$ be a maximal abelian subspace of $\mathfrak{p}$ such that $m(y) \in \mathfrak{a}$. Since $x$ and $y$ are in the same $G$-orbit, there exists $g \in G$ such that $x=g y$. If $A$ is the analytic subgroup of $G$ corresponding to the subalgebra $\mathfrak{a}$ of $\mathfrak{g}$, the global Cartan decomposition of $G$ with respect to a reads $G=K A K$. In particular, there exist $k_{1}, k_{2} \in K$ and $Z \in \mathfrak{a}$ such that $g=k_{1} \exp Z k_{2}$ i.e, $x=k_{1} \exp Z k_{2} y$. Since $m(k x)=A d(k) m(x), x$ is fixed by $m(x)$ if and only if $k x$ is fixed by $m(k x)$ i.e., $x$ is a critical point of $\|m\|^{2}$ together with its entire $K$-orbit. We may therefore assume that $k_{1}=k_{2}=1$. Consider the function $g: \mathbb{R} \longrightarrow \mathbb{R}$, given by 
$g(t)=m_{m(y)}(\exp t Z y)$. If $w$ is a representative of $y$, then according to eq.(1),

(3) $g(t)=\left.\frac{1}{2\|\exp t Z w\|^{2}} \frac{d}{d s}\right|_{s=0}\|\exp \operatorname{sm}(y) \exp t Z w\|^{2}$

$$
=\left.\frac{1}{2\|\exp t Z w\|^{2}} \frac{d}{d s}\right|_{s=0}\|\exp t Z \exp s m(y) w\|^{2} .
$$

Since $y$ is a critical point of $\|m\|^{2}$, it is fixed by $\exp t m(y)$, therefore $w$ is an eigenvector of $m(y)$ with some real eigenvalue $a \cdot a=\frac{(m(y) w, w)}{(w, w)}=m_{m(y)}(y)=$ $\|m(y)\|^{2}$. Eq.(3) implies now that $g(t)=a=\|m\|^{2}(y)$. Also for future reference we record what we have just established.

If $y$ is a critical point of $\|m\|^{2}$ and $Z \in \mathfrak{p}$ commutes with $m(y)$, then the function

$$
g: \mathbb{R} \longrightarrow \mathbb{R}, g(t)=m_{m(y)}(\exp t Z y) \text { is constant, and } g(t)=\|m\|^{2}(y) .
$$

In particular, we have that $g(0)=g(1)$ i.e., $m_{m(y)}(x)=m_{m(y)}(y)$. If $m(y) \neq 0$ it follows that $m_{m(y)}(x) \neq 0$, hence $m(x) \neq 0$, a contradiction.

We shall now restrict ourselves to the situation of a critical point $x$ such that $m(x) \neq 0$, and shall prove in this case also that for all critical points $y \in G x$, $\|m\|^{2}(y)=\|m\|^{2}(x)$.

To begin, define $\mu: V \times \mathfrak{p} \longrightarrow \mathbb{R}$, by letting $\mu(v, \beta)$ be the smallest eigenvalue of $\beta$ such that the projection of $v$ onto the corresponding eigenspace is nonzero. Note that for $g \in G, \mu(g v, A d g(\beta))=\mu(v, \beta)$. (Indeed, this amounts to saying that $v$ is an eigenvector of $\exp \beta$ with eigenvalue $a$ if and only if $g v$ is an eigenvector of $g \exp \beta g^{-1}$ with eigenvalue $a$.) Define

$$
M: \mathbb{P}(V) \longrightarrow \mathbb{R} \text {, by } M(x)=\sup _{\beta \in \mathfrak{p}-\{0\}} \frac{\mu(v, \beta)}{\|\beta\|} \text {, where } v \text { represents } x .
$$

Lemma 3. Assume that $m(x) \neq 0$. Then the following are equivalent:

(i) $x$ is a critical point of $\|m\|^{2}: \mathbb{P}(V) \longrightarrow \mathbb{R}$.

(ii) $M(x)=\|m(x)\|>0$.

Proof. Let $v \in V$ represent $x$, and let $v=\sum_{i} v_{i}$ be a decomposition of $v$ according to eigenspaces for $\beta \in \mathfrak{p}: \beta v=\sum_{i} a_{i} v_{i}$. The $a_{i}$ s are distinct eigenvalues of $\beta$ and the $v_{i}$ s are assumed to have unit norm. Then

$$
m_{\beta}(x)=\frac{1}{\|v\|^{2}} \sum_{i=1}^{n} a_{i}\left\|v_{i}\right\|^{2} \geq \text { smallest } a_{i}, \text { so } \mu(v, \beta) \leq m_{\beta}(x) .
$$

For arbitrary $\beta$ in $\mathfrak{p}, \frac{(m(x), \beta)}{\|\beta\|} \leq\|m(x)\|$, hence $\frac{m_{\beta}(x)}{\|\beta\|} \leq\|m\|(x)$. But by eq. (5), for arbitrary $\beta \in \mathfrak{p}, \mu(v, \beta) \leq m_{\beta}(x)$. Thus for all $\beta \in \mathfrak{p}, \frac{\mu(x, \beta)}{\|\beta\|} \leq\|m\|(x)$, 
therefore

$$
M(x) \leq\|m\|(x)
$$

Equality holds in eq. (5) if and only if $v$ over $x$ is in an eigenspace of $\beta$, if and only if $d m_{\beta}(x)=0$. Hence $d m_{m(x)}=0$ if and only if $\mu(v, m(x))=m_{m(x)}(x)=$ $\|m(x)\|^{2}$ if and only if $\frac{\mu(v, m(x))}{\|m(x)\|}=\|m\|(x)$. In conjunction with eq. (6), since by definition $M(x) \geq \frac{\mu(v, m(x))}{\|m(x)\|}$, this shows that the condition $M(x)=\|m\|(x)$ is equivalent to $v$ over $x$ being an eigenvector of $m(x)$, and proves Lemma 3 .

For the purposes of the following lemma, we consider $M: V \longrightarrow \mathbb{R}$. Recall that $M(v)=\sup _{\beta \in \mathfrak{p}-\{0\}} \frac{\mu(v, \beta)}{\|\beta\|}$.

Lemma 4. 1. For every $v$ in $V$ there exists $\beta \in \mathfrak{p}$ such that $M(v)=\frac{\mu(v, \beta)}{\|\beta\|}$.

2. $M$ assumes finitely many values.

3. $M$ is constant on $G$-orbits.

Proof. Let $\mathfrak{a}$ be a maximal abelian subspace of $\mathfrak{p}$. There is a finite set $\Lambda \subset \mathfrak{a}^{\star}$ and a decomposition of $V, V=\oplus_{\lambda \in \Lambda} V_{\lambda}$ such that the spaces $V_{\lambda}$ are orthogonal and $v \in V_{\lambda}$ if and only if $\forall \beta \in \mathfrak{a}, \beta v=\lambda(\beta) v$. Consider the functions $\mu_{v}: \mathfrak{a} \longrightarrow \mathbb{R}, \mu_{v}(\beta)=\mu(v, \beta)$. For a fixed $v$, there is a unique decomposition $v=\sum_{i=1}^{k} v_{\lambda_{i}}, v_{\lambda_{i}} \in V_{\lambda_{i}}$. Then $\mu_{v}(\beta)=\min _{1 \leq i \leq k}\left\{\lambda_{1}(\beta), \ldots, \lambda_{k}(\beta)\right\}$. Since there are finitely many patterns of decomposition of $v$ according to the weight spaces $V_{\lambda}$, as $v$ ranges through $V$, there are finitely many functions $\mu_{v}$. Also, $\mu_{v}: \mathfrak{a} \longrightarrow \mathbb{R}$ is continuous as the pointwise minimum of continuous functions. Therefore the function $M_{v}^{\mathfrak{a}}: \mathfrak{a} \longrightarrow \mathbb{R}, M_{v}^{\mathfrak{a}}(\beta)=\frac{\mu(v, \beta)}{\|\beta\|}$ attains its maximum. For each $v$ the function $M^{\mathfrak{a}}: V \longrightarrow \mathbb{R}, M^{\mathfrak{a}}(v)=\sup _{\beta \in \mathfrak{a}-\{0\}} \frac{\mu(v, \beta)}{\|\beta\|}$ is actually given by $\frac{\mu(v, H)}{\|H\|}$ for some $H$ in $\mathfrak{a}$. Moreover, as there are only finitely many functions $\mu_{v}: \mathfrak{a} \longrightarrow \mathbb{R}$ as $v$ ranges through $V, M^{\mathfrak{a}}$ assumes finitely many values.

The maximal abelian subspaces of $\mathfrak{p}$ are conjugate under $A d K$, and given such a subspace $\mathfrak{a} \subset \mathfrak{p}, \mathfrak{p}=\bigcup_{k \in K} A d(k)(\mathfrak{a})$. Therefore

$$
\begin{aligned}
M(v) & =\sup _{\beta \in \mathfrak{p}} \frac{\mu(v, \beta)}{\|\beta\|}=\sup _{k \in K} \sup _{\beta \in \mathfrak{a}} \frac{\mu(v, A d(k) \beta)}{\|A d(k) \beta\|} \\
& =\sup _{k \in K} \sup _{\beta \in \mathfrak{a}} \frac{\mu(k v, \beta)}{\|\beta\|}=\sup _{k \in K} M^{\mathfrak{a}}(k v)=\max _{k \in K} M^{\mathfrak{a}}(k v) \\
& =\frac{\mu(k v, \beta)}{\|\beta\|}, \text { for some } k \in K, \beta \in \mathfrak{a} \\
& =\frac{\mu(v, \alpha)}{\|\alpha\|}, \text { for } \alpha=A d k^{-1}(\beta) \in \mathfrak{p} .
\end{aligned}
$$


This proves Part 1 of the lemma, and also Part 2, since for each $v, M(v)$ is chosen from a subset of a fixed finite set of values.

For Part 3, it is enough to show that for all $v$ in $V$ and $g$ in $G, M(v) \geq M(g v)$. Fix a maximal abelian subspace $\mathfrak{a} \subset \mathfrak{p}$. Then, notations being as in the proof of Part 1,

$$
M(v)=\max _{k \in K} M^{\mathfrak{a}}(k v) .
$$

In order to prove that $M(v) \geq M(g v)$ holds in general, it is enough to show that for all $v \in V, g \in G$, there exists $k \in K$ such that $M^{\mathfrak{a}}(k v) \geq M^{\mathfrak{a}}(g v)$. Fix arbitrary $v$ and $g$. Let $H \in S^{\operatorname{dim} \mathfrak{a}-1} \subset \mathfrak{a}$ be such that $M^{\mathfrak{a}}(g v)=M_{g v}^{\mathfrak{a}}(H)$ i.e., $H$ is the maximum of $\mu_{g v}$ restricted to the unit sphere in $\mathfrak{a}$. We want to define a convenient ordered orthonormal basis for $V$. We define first an order on the set of weights $\Lambda$, then we choose orthonormal bases for each of the weight spaces $V_{\lambda}$ and put them together to form an ordered orthonormal basis for $V$ in the following way: if $\lambda_{1}, \lambda_{2} \in \Lambda, \lambda_{1} \leq \lambda_{2}$, then the vectors in the basis for $V_{\lambda_{1}}$ precede those in the basis for $V_{\lambda_{2}}$ in the ordered basis for $V$. (The order of the vectors that form a basis for one weight space is irrelevant and can be chosen arbitrarily.) Now the order on weights is established as follows. Let $\Lambda_{0}=\left\{\lambda \in \Lambda\right.$ such that the orthogonal projection of $g v$ on $V_{\lambda}$ is 0$\}$ and let $\Lambda_{1}=\Lambda \backslash \Lambda_{0}$. Choose an arbitrary order on $\Lambda_{0}$ and declare the elements of $\Lambda_{0}$ to be less than those of $\Lambda_{1}$. Order the elements of $\Lambda_{1}$ according to the order of their evaluation at $H$ defined above, i.e., for $\lambda, \tilde{\lambda} \in \Lambda, \lambda \leq \tilde{\lambda}$ if $\lambda(H) \leq \tilde{\lambda}(H)$. Let $g v=\sum_{i \geq i_{0}} a_{i} v_{i}$, where $\left\{v_{1}, \ldots, v_{n}\right\}$ is the ordered basis of $V$ just obtained and $a_{i} \neq 0$. Also, for future convenience, let $\tilde{\lambda}$ be the weight such that $v_{i_{0}} \in V_{\tilde{\lambda}}$ that is, $\tilde{\lambda}$ is the smallest element of $\Lambda_{1}$. According to an Iwasawa decomposition of the image $\tilde{G}$ of $G$ in $G L(n, \mathbb{R})$, it is possible to write $g=n a k$ for some $n, a, k \in \tilde{G}$ such that $n$ is lower triangular with 1 s on the diagonal, $a$ is diagonal with positive diagonal entries and $k$ is orthogonal. Then $k v=a^{-1} n^{-1} g v$. Since $n$ is lower triangular and $a$ is diagonal, it is clear that $k v$ has a nonzero projection onto $v_{i_{0}}$ and no nontrivial projections onto the vectors that precede it in the ordered basis of $V$. Thus

$$
M_{k v}^{\mathfrak{a}}(H)=\tilde{\lambda}(H)=M_{g v}^{\mathfrak{a}}(H)=M^{\mathfrak{a}}(g v),
$$

hence

$$
M^{\mathfrak{a}}(k v)=\sup _{X \in S^{\operatorname{dim} \mathfrak{a}-1} \subset \mathfrak{a}} M_{k v}^{\mathfrak{a}}(X) \geq M_{k v}^{\mathfrak{a}}(H) \geq M^{\mathfrak{a}}(g v) .
$$

This establishes Part 3 of Lemma 4.

The first part of the theorem follows very quickly now. If $x$ is a critical point of $\|m\|^{2}, m(x) \neq 0$, then by Lemma $3,\|m(x)\|=M(x)$. According to eq. (6) in the proof of the same lemma, $\|m(y)\| \geq M(y)$ for arbitrary $y$. Hence for arbitrary $g,\|m(g x)\| \geq M(g x)=M(x)=\|m(x)\|$ i.e., $x$ is a minimum of $\left.\|m\|^{2}\right|_{G x}$. Moreover, if $y$ is another critical point of $\|m\|^{2}$ in the same $G$-orbit, 
$y=g x$ for some $g \in G$, then

$$
\|m(y)\|=M(y)=M(g x)=M(x)=\|m(x)\|,
$$

which will be very important in establishing the second part of the theorem. The last ingredient needed before proceeding to do this is the following

Lemma 5. Let $\alpha$ be in $\mathfrak{p}, \alpha \neq 0$, and $x \in \mathbb{P}(V)$ such that $x$ is not fixed by $\alpha$. Then $f: \mathbb{R} \longrightarrow \mathbb{R}, f(t)=m_{\alpha}(\exp (t \alpha) x)$ is a strictly increasing function.

Proof. We show that the derivative of $f$ is positive. Let $v \in V$ represent $x$ and let $v=\sum_{i} v_{i}$ be a decomposition of $v$ according to eigenspaces of $\alpha, \alpha v_{i}=a_{i} v_{i}$. Then $\exp (t \alpha) v=\sum_{i} e^{t a_{i}} v_{i}$, so

$$
f(t)=m_{\alpha}(\exp (t \alpha) x)=\frac{\sum a_{i} e^{2 t a_{i}}\left\|v_{i}\right\|^{2}}{\sum e^{2 t a_{i}}\left\|v_{i}\right\|^{2}}
$$

hence

$$
f^{\prime}(t)=\frac{2 \sum_{i<j}\left(a_{i}-a_{j}\right)^{2} e^{2 t\left(a_{i}+a_{j}\right)}\left\|v_{i}\right\|^{2}\left\|v_{j}\right\|^{2}}{\left(\sum e^{2 t a_{i}}\left\|v_{i}\right\|^{2}\right)^{2}} .
$$

Since $v$ is not an eigenvector of $\alpha$, at least two distinct eigenvalues $a_{i}$ of $\alpha$ appear in the above expression of $f^{\prime}(t)$, hence $f^{\prime}(t)$, clearly nonnegative, also never vanishes.

Let us now prove Part 2 of the theorem. Let $x$ and $y$ be critical points of $\|m\|^{2}$ such that $y=g x$ for some $g \in G$ and $\|m\|^{2}(x) \neq 0$. Then, as noted above, Lemmas 3 and 4 imply that $\|m\|^{2}(x)=\|m\|^{2}(y)$. Let $\mathfrak{a} \subset \mathfrak{p}$ be a maximal abelian subspace containing $m(x)$. Let $A$ be the analytic subgroup of $G$ corresponding to $\mathfrak{a}$. We use a global Cartan decomposition to write $G=K A K$. In particular, it is possible to write $g=k_{1} \exp \beta k_{2}$, for $k_{1}, k_{2} \in K$ and $\beta \in \mathfrak{a}$. Thus $y=k_{1} \exp \beta k_{2} x$. As noted before, due to the $K$-equivariance of $m, x$ is a critical point of $\|m\|^{2}$ together with its entire $K$-orbit, therefore it can be assumed without loss of generality that $y=\exp \beta x$. Moreover, since $m(x)$ fixes $x$ and commutes with $\beta$, it can be assumed, also without loss of generality, that $m(x)$ and $\beta$ are orthogonal. We want to show: if $\exp \beta \neq 1$, then $\|m\|^{2}(y)>\|m\|^{2}(x)$, a contradiction.

Eq. (4) has established the constancy of $g(t)=m_{m(x)}(\exp t \beta x)$. In particular it implies that $m_{m(x)}(y)=m_{m(x)}(\exp \beta x)=m_{m(x)}(x)=\|m\|^{2}(x)$. Since $m(x)$ and $\beta$ are orthogonal,

$$
\|m\|^{2}(y) \geq \frac{(m(y), m(x))^{2}}{\|m(x)\|^{2}}+\frac{(m(y), \beta)^{2}}{\|\beta\|^{2}}=\|m\|^{2}(x)+\frac{m_{\beta}(\exp \beta x)^{2}}{\|\beta\|^{2}} .
$$

By Lemma 5 , if $\beta \neq 0$, the function $f(t)=m_{\beta}(\exp t \beta x)$ is strictly increasing. $f(0)=m_{\beta}(x)=0$, since $m(x)$ and $\beta$ are orthogonal, and $f(1)=m_{\beta}(\exp \beta x)$. We conclude that $\|m\|^{2}(y)>\|m\|^{2}(x)$, a contradiction. Therefore $\beta=0$ i.e., $x$ and $y$ are in the same $K$-orbit. 


\section{Acknowledgement}

I am grateful to Professor Wilfried Schmid for guiding me through this problem, as well as for his numerous encouragements and generous concern.

\section{References}

[1] V. Guillemin and S. Sternberg, Convexity properties of the moment mapping, Invent. Math. 67 (1982), no. 3, 491-513.

[2] T.H. Koornwinder (ed.), The Structure of Real Semisimple Lie Groups, Matematisch Centrum, Amsterdam 1982.

[3] I. Mirković, T. Uzawa, and K. Vilonen, Matsuki correspondence for sheaves, Invent. Math. 109 (1992), no. 2, 231-245.

[4] D. Mumford, Geometric Invariant Theory, Springer Verlag, New York 1965.

[5] L. Ness, A stratification of the null cone via the moment map, Amer. J. Math. 106 (1984), no. $6,1281-1325$.

[6] L. Ness, Mumford's numerical function and stable projective hypersurfaces, Springer Lecture Notes \#732 Algebraic Geometry, Proceedings, Copenhagen 1978, 417-454.

[7] W. Schmid and K. Vilonen, On the geometry of nilpotent orbits, Asian J. Math. 3 (1999), no. 1, 233-274.

[8] J. Sekiguchi, Remarks on nilpotent orbits of a symmetric pair, J. Math. Soc. Japan 39 (1987), no. 1, 127-138.

Harvard University, Department of Mathematics, One Oxford Street, Cambridge, MA 02138, USA.

E-mail address: marian@math.harvard.edu 